Schmerz Nachr 2022 $22: 1$

https://doi.org/10.1007/s44180-022-00017-y

Angenommen: 7. Februar 2022

Online publiziert: 24. Februar 2022

(c) The Author(s), under exclusive licence to Springer-Verlag GmbH Austria, ein Teil von Springer Nature 2022

\title{
Schmerzausbildung erweitern und vertiefen
}

Rudolf Likar

Abteilung für Anästhesiologie und Intensivmedizin, Klinikum Klagenfurt, Klagenfurt, Österreich

Liebe Leser*innen, liebe Kolleg*innen!

Ein Thema, das uns in der Österreichischen Schmerzgesellschaft insgesamt und mich im Speziellen seit Jahren intensiv beschäftigt, ist die weitere Verbesserung und Erweiterung der Schmerzausbildung für unsere Kolleginnen und Kollegen.

Die schmerzmedizinische Basisausbildung mit Abschluss des Schmerzdiploms haben bisher 1424 Ärzt*innen erfolgreich absolviert. Mehr als ein Drittel davon entfällt auf Kolleg*innen aus dem Anästhesiebereich, ein knappes Drittel auf Allgemeinmediziner*innen. Das verbleibende Drittel verteilt sich auf Neurolog*innen (150) und andere Fachbereiche. An dieser Verteilung kann man gut erkennen, dass es hier in manchen Fachbereichen noch durchaus Luft nach oben gibt.

Neben dieser Basisausbildung sehen wir eine weiterführende, vertiefende Spezialausbildung als notwendiges zusätzliches Ausbildungselement an, um die Schmerzkompetenz innerhalb der Ärzteschaft zu verbessern. Um etwa die im Qualitätsstandard Unspezifischer Rückenschmerz vorgesehene abgestufte Versorgung mit Basisversorgung, spezialisierte Versorgung und hochspezialisierte Versorgung flächendeckend anbieten zu können, braucht es noch viel mehr gut ausgebildete Schmerzmediziner*innen, als sie derzeit zur Verfügung stehen.

Ein entsprechendes Konzept für eine solche vertiefende Spezialausbildung haben wir bereits erarbeitet, es liegt längst am Tisch. Das Programm umfasst $80 \mathrm{~h}$ Theorie plus $400 \mathrm{~h}$ Praxis.

Bisher konnte in mehreren Gesprächsrunden mit der Ärztekammer leider noch kein Konsens für die Implementierung des neuen Ausbildungstools erreicht werden. Am 1. April findet bekanntermaßen die
Ärztekammerwahl 2022 statt. Nachdem sich die neu gewählten Gremien danach wieder konstituiert haben, werden wir seitens des ÖSG-Vorstandes einen neuerlichen Anlauf unternehmen, um das Thema voranzutreiben, um in absehbarer Zeit hoffentlich zu einem positiven Abschluss zu kommen. Wir bleiben dran!

Ihr

Rudolf Likar

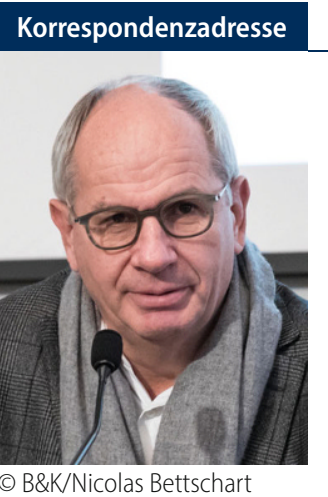

Prim. Univ.-Prof. Dr. Rudolf Likar, MSc

Abteilung für Anästhesiologie und Intensivmedizin, Klinikum Klagenfurt Klagenfurt, Österreich rudolf.likar@kabeg.at

Prim. Univ.-Prof. Dr. Rudolf Likar, Schriftleitung der Schmerz Nachrichten, Generalsekretär der ÖSG, Vorsitzender der Sektion Schmerz in der ÖGARI; Vorsitzender Anästhesie Nachrichten Advisory Board; Past President und Generalsekretär der ÖGARI, Past President und erster Vizepräsident der OPG; Vorstand der Abteilung für Anästhesiologie und Intensivmedizin, Klinikum Klagenfurt.

Interessenkonflikt. R. Likar gibt an, dass kein Interessenkonflikt besteht.

Hinweis des Verlags. Der Verlag bleibt in Hinblick auf geografische Zuordnungen und Gebietsbezeichnungen in veröffentlichten Karten und Institutsadressen neutral. 